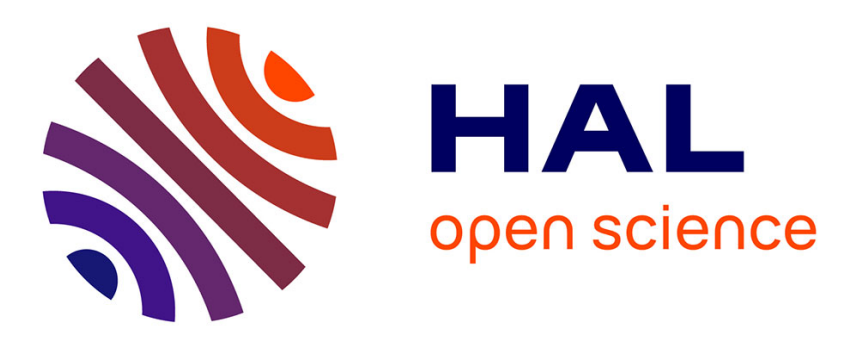

\title{
Macroscopic control of high-order harmonics quantum-path components for the generation of attosecond pulses
}

\author{
H. Merdji, M. Kovačev, W. Boutu, P. Salieres, F. Vernay, B. Carré
}

\section{- To cite this version:}

H. Merdji, M. Kovačev, W. Boutu, P. Salieres, F. Vernay, et al.. Macroscopic control of high-order harmonics quantum-path components for the generation of attosecond pulses. Physical Review A : Atomic, molecular, and optical physics [1990-2015], 2006, 74 (4), 10.1103/PhysRevA.74.043804 . hal02145556

\section{HAL Id: hal-02145556 https://hal.science/hal-02145556}

Submitted on 3 Jun 2019

HAL is a multi-disciplinary open access archive for the deposit and dissemination of scientific research documents, whether they are published or not. The documents may come from teaching and research institutions in France or abroad, or from public or private research centers.
L'archive ouverte pluridisciplinaire HAL, est destinée au dépôt et à la diffusion de documents scientifiques de niveau recherche, publiés ou non, émanant des établissements d'enseignement et de recherche français ou étrangers, des laboratoires publics ou privés. 


\title{
Macroscopic control of high-order harmonics quantum-path components for the generation of attosecond pulses
}

\author{
H. Merdji, ${ }^{1}$ M. Kovačev, ${ }^{2}$ W. Boutu, ${ }^{1}$ P. Salières, ${ }^{1}$ F. Vernay,,${ }^{1}$ and B. Carré ${ }^{1}$ \\ ${ }^{1}$ CEA/DSM/DRECAM/Service des Photons, Atomes et Molécules, Bât. 522, CEA-Saclay, 91191 Gif-sur-Yvette, France \\ ${ }^{2}$ Institut für Quantenoptik, Leibniz. Universität Hannover, Welfengarten 1, D-30167 Hannover, Germany
}

(Received 22 March 2006; published 4 October 2006)

\begin{abstract}
We present measurements of the different quantum path contributions to the high-order harmonic emission. Through spatial and spectral filtering, we evidence the strong correlation between the spatial and spectral distributions, which allows us to quantify the contribution of each quantum path. A systematic analysis as a function of the generating parameters has been done to identify the conditions for efficient generation and selection of a single quantum path. We show that combining phase matching and spatial filtering allows maximizing and selecting the short quantum path contribution, condition for the generation of "clean" and intense attosecond pulses.
\end{abstract}

DOI: 10.1103/PhysRevA.74.043804

PACS number(s): 42.65.Ky, 42.65.Re, 42.87.Bg

\section{INTRODUCTION}

The generation of the high-order harmonics (HHG) of intense laser pulses focused in gases is attracting much attention due to both its fundamental and applied interest $[1,2]$. Indeed, the advanced characterization of the process both gives insight into the ultrafast atomic dynamics in the laser field, and qualify this source of XUV radiation for potential applications. In particular, measurements of the spatial [3-7] and temporal [8-11] coherence properties have allowed a deeper understanding of the generation process, and have revealed their good quality, which is unique in this spectral range. Together with the short pulse duration and the high energy (up to microjoule energy per pulse $[12,13]$ ), these properties have found applications in pump-probe experiments, e.g., for interferometry in plasma physics $[14,15]$, but also in solid state physics and in atomic and molecular spectroscopy [16]. The good coherence also makes the HHG source particularly suitable for seeding soft-x-ray lasers [17] or free-electron lasers [18]. Finally, the spectral coherence over the extended harmonic spectrum is the key for the generation of attosecond pulses: the high harmonic source has opened the field of the so-called attoscience which has been spectacularly growing in the last few years (for a review, see Ref. [19]).

The major above breakthroughs rely on the deep theoretical understanding and experimental mastery of HHG. Profound insight in the generation process has been provided by the semiclassical three-step model $[20,21]$, in which an electron first tunnels out of the atomic potential, is then accelerated in the laser field and finally driven back to the ion leading to recombination on the ground state with emission of a burst of XUV light. Quantum description of HHG within SFA [22] and TDSE [23-25], as well as direct experimental probe of the electron dynamics [26], have grounded the notion of electron trajectories in the laser field, the so-called quantum paths. For harmonic of $q$ th order in the plateau, mainly two quantum paths contribute to the radiating dipole $D_{q}$. The two paths differ by the ionization time $t_{i}$ and emission time $t_{e}$, or equivalently the travel time $\tau=t_{e}-t_{i}$ of the electron wave packet in the continuum. For the so-called "short" trajectory (index $j=1$ ), the travel time $\tau_{1}$ is of the order of half the optical period, whereas for the "long" trajectory $(j=2), \tau_{2}$ is close to the optical period. The dipole $D_{q}$ (equivalently the local nonlinear polarization) can thus be expressed as the sum $D_{q}=D_{1} e^{i \phi_{1}}+D_{2} e^{i \phi_{2}}$ of the two contributions. In the Lewenstein model [22], the phase $\phi_{j}$ identifies to the quasiclassical action along the $j$ path and therefore depends on the parameters $t_{e j}, \tau_{j}$, laser intensity $I$ and XUV frequency $q$ (the order $q$ is considered as a continuous variable denoting the XUV frequency in $\omega$ unit). Note that, ultimately, the times $t_{e j}, \tau_{j}$ are in turn functions of $I$ and $q$, so that for each class of trajectories the phase $\phi_{j}$ is completely determined by the laser intensity and the harmonic order $q$. One further demonstrates that the phase differential can be written as $d \phi_{j}=\alpha_{j} d I+t_{e j} d q$, where the partial derivative $\alpha_{j}$ is determined by the travel time $\tau_{j}$ and is therefore larger for the long trajectories than for the short ones. The $\alpha$ and $t_{e}$ coefficients vary slowly with the frequency $q$. In the cutoff, the two classes of trajectories merge into one.

After propagation, the macroscopic XUV field in the plateau is the sum of two terms, refered to as $\tau_{1^{-}}$and $\tau_{2}$-contributions, respectively,

$$
E_{q}=E_{1} e^{i \Phi_{1}}+E_{2} e^{i \Phi_{2}}
$$

If we assume conditions close to phase-matching, the macroscopic phases take the simple form $\Phi_{j} \approx q \phi_{L}+\phi_{j}$, where $\phi_{L}$ is the laser phase including the $-\omega t$ term. On the one hand, the $q$ dependence of the phases in Eq. (1) has important consequences for the generation of attosecond pulses when they are obtained as a coherent sum of quasi-phaselocked (discrete harmonic or continuous) components $E_{q}$ in the plateau [26-28]. On the other hand, the $I$ dependence, i.e., the temporal and spatial variations, of the phases $\Phi_{j}\left(\phi_{j}\right)$ determines the coherence properties of $E_{q}$, and in particular its spectral and spatial characteristics [1]. First, it is responsible for the intrinsic chirp of the harmonic emission. Second, it also conditions the spatial phase and therefore the spatial profile of the XUV field. The different $I$ dependence of the two quantum paths components (different $\alpha_{j}$ ) thus leads to a spatial and spectral separation of their contribu- 
tions to the macroscopic harmonic field [24,29]. The separation has been used to study their different temporal coherence $[8,9]$ and phase matching [10]. However, to our knowledge, there has been no experimental study of the amplitudes $E_{1}$ and $E_{2}$, i.e., of the relative weights of the two contributions.

The discrimination and control of the $\tau_{1}$ and $\tau_{2}$ contributions are of great importance, first for a fundamental insight on HHG and second for controlling the XUV emission. Theoretically, the relative weight of the $\tau_{1^{-}}$and $\tau_{2}$-contributions to the single atom response (i.e., $D_{1} / D_{2}$ ) is still somewhat controversial: the SFA and TDSE approaches lead to different predictions, the $\tau_{2}$ contribution being usually larger in SFA (and presumably overestimated) than in TDSE $[24,25]$. Experimental studies are thus needed. Note that the relative weight of the macroscopic contributions $\left(E_{1} / E_{2}\right)$ is also affected by the propagation, i.e., by the way phase matching is achieved for each contribution. On the control side, producing an XUV field $E_{q}$ at given frequency, with definite spectral and spatial characteristics, implies in general that only one $E_{j}$ contribution is selected in Eq. (1), thus limiting the space and time phase variation. Moreover, the production of attosecond pulses from emission in the plateau demands to select one single quantum-path, the condition for synchronized (phase-locked) emission over a broad spectral range [30-34]. In the case where the two classes of trajectories contribute, two bursts are emitted with different timings every half cycle, which blurs the attosecond structure. In a number of recent works $[26,27,32,33,35,36]$ the subcycle pulses in HHG have been characterized for a set of harmonics - up to 30 harmonics in Ref. [26]—from measurement of the harmonic relative phases $\left(d \phi_{j}=t_{e j} d q\right.$ at fixed I). They have revealed the possibility to generate pulses as short as 130 as that could be further compressed after compensation of their intrinsic chirp $[26,33,37]$. The experiments assume and partially confirm that, for appropriate generation conditions, propagation and spatial filtering selects mainly the contribution of one quantum path (namely, the "short" path). Actually, more accurate characterization of the subcycle electron dynamics and even shorter attosecond pulses could be achieved from a direct monitoring and a more complete discrimination of the paths contributions.

In this paper, we present measurements of the contributions of the two quantum paths. The control/discrimination of the $\tau_{1}$ and $\tau_{2}$ contributions can be envisaged at two levels. At the "upstream" level, one favors one particular contribution by choosing the appropriate conditions in the generation process itself. This is made possible by adjusting the focussing geometry of the laser beam, then using propagation and phase-matching as a "filter." At the "downstream" level, one takes advantage of the different spectral and spatial characteristics of the $E_{1}$ and $E_{2}$ components to discriminate them through spectral or spatial filtering. We demonstrate the possibility of combining upstream and downstream control of the $\tau_{1}$ and $\tau_{2}$ contributions. In the downstream control, we show in turn how the filtering in the spatial domain can be consistently combined with the one in the spectral domain, and the relative weight of the $\tau_{1}$ and $\tau_{2}$ contributions estimated. Finally, we study the variation of this relative weight as a function of different generation parameters.

\section{EXPERIMENT}

\section{A. Identification of path contributions}

We first explain the principle of the upstream and downstream controls of the $\tau_{1}$ and $\tau_{2}$ components. As mentioned, it is based on the different phase properties of the $\tau_{1}$ and $\tau_{2}$ contributions to the atomic dipole for the upstream control, to the related macroscopic field for the downstream control. Upstream control involves the spatial characteristic of the dipole phase $\phi_{j}$. In the medium, the macroscopic field $E_{q}$ builds up along propagation under the phase-matching condition [38]

$$
\vec{\nabla} \Phi_{j} \approx \vec{\nabla}\left(q \phi_{L}+\phi_{j}\right)
$$

i.e., equality of the wave vectors of the harmonic field and nonlinear polarization, where $\vec{\nabla} \phi_{j}=\alpha_{j} \vec{\nabla} I$. In the case of a free propagating laser beam focused in a gas jet, it has been demonstrated that the smaller gradient $\alpha_{1} \vec{\nabla} I$ for the short trajectory in Eq. (2) $\left(\alpha_{1}<\alpha_{2}\right)$ determines its dominant contribution to on-axis emission, when the laser beam is focused before the jet $[24,29,38]$. Conversely, the large gradient $\alpha_{2} \vec{\nabla} I$ for the long trajectory determines its dominant contribution to off-axis emission, when the laser beam is focused after the jet. The two contributions are therefore discriminated upstream through the focussing geometry. When the two contributions are significantly present after propagation, they are also separated downstream in the spatial domain, in the form of an inner and an outer region in the far-field profile.

Together with the spatial discrimination, the two contributions are discriminated downstream in the spectral domain. Hence in a real laser pulse where $I$ varies in time (within adiabatic approximation for $\mathrm{HHG}$ ), the intensitydependent term in the phase $\Phi_{j}\left(\phi_{j}\right)$ causes a frequency modulation, a chirp, of the harmonic emission [29]:

$$
\Delta \omega(t)=-\frac{\partial \Phi_{j}(t)}{\partial t}=-\alpha_{j} \frac{\partial I(t)}{\partial t} .
$$

It is clear from Eq. (3) that the spectral width $\Delta_{1}$ [proportional to $\left.\left(\partial \Phi_{1} / \partial t\right) \max \right]$ of the $E_{1}$ contribution in Eq. (1) will be smaller than that of $E_{2}$. Thus, spectral or spatial filtering of the macroscopic harmonic field should allow a downstream selection of either $\tau_{1}$ or $\tau_{2}$ contribution.

In a first experiment, we perform a similar analysis as in Refs. $[8,9]$ in order to identify the two contributions according to the above discrimination in the spectral and spatial domains. The experiment was carried out at the femtosecond LUCA laser facility of CEA/DRECAM in Saclay (titanium:sapphire system at $800 \mathrm{~nm}, 80 \mathrm{~mJ}, 60 \mathrm{fs}, 20 \mathrm{~Hz}$ ). The laser energy is adjusted using a diaphragm. Two phase-locked IR pulses of $\sim 1 \mathrm{~mJ}$ energy are produced in a Michelson type interferometer and focused with a $1 \mathrm{~m}$ focal length lens $4 \mathrm{~mm}$ after an argon jet (1 mm length) at a backing pressure of 900 Torr. The interferometer is identical to the one used in Refs. $[39,40]$. It allows for generating two phase-locked harmonic pulses separated in space and delayed in time, which allows for delay-dependent interference in the far-field. As shown in Fig. 1, XUV light is analyzed with a monochromator consisting in a toroidal mirror, a grating, and an exit 


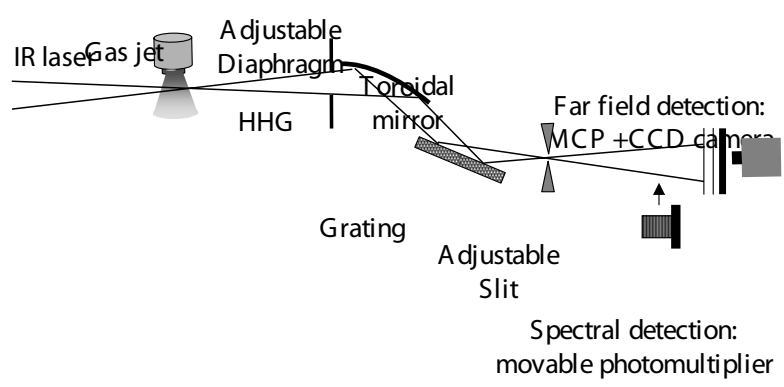

FIG. 1. Experimental setup for the spectral and spatial analysis of quantum path contributions.

slit. The width of the slit can be adjusted to tune the spectral acceptance from few nanometers (selection of one harmonic order with full spectral profile transmitted) to $0.05 \mathrm{~nm}$ (harmonic spectral analysis). The far-field spatial distribution is measured on microchannel plates coupled to a phosphor screen and a 12 bit-CD camera. Under assumption of good mutual coherence of the two harmonic sources, the temporal coherence can be mapped in the beam cross section from the far field interference pattern, by varying the delay between the two pulses (laser pulses delay in the Michelson). The slit in the spectral plane was adjusted to select a single harmonic order. We will consider in this study the 17th harmonic which is representative of the behavior in the plateau region. In the inset in Fig. 2, the far-field interference pattern for 17 th harmonic, measured in single shot and at zero delay between the two pulses, clearly reveals the two regions that we associate to the contributions of the short (inner region for on-axis emission) and long trajectory (outer region for off-axis emission). The fringes visibility defined as $\left(I_{\max }-I_{\min }\right) /\left(I_{\max }+I_{\min }\right)$ is plotted in Fig. 2 as a function of

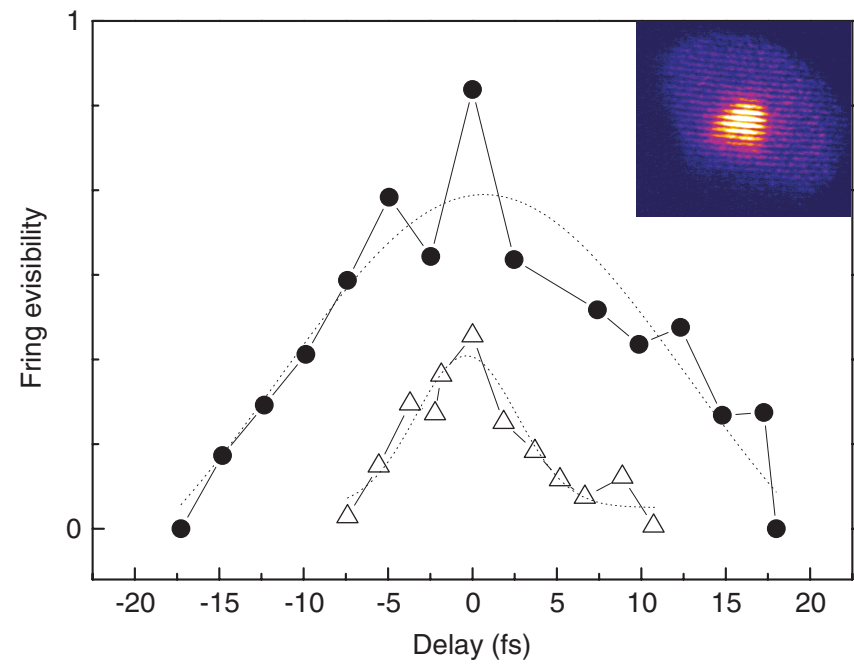

FIG. 2. (Color online) Average visibility of the fringes in the far-field interference pattern for the 17th harmonic, generated in argon, as a function of delay (1st-order autocorrelation trace): short (full circle) and long (open triangle) trajectories contributions. In dotted line are shown the Gaussian fits of the measured traces. The FWHM gives the coherence time. Inset: Interference pattern recorded at 0 delay. the delay, for the inner and outer regions. Because of the highly controlled, coherent physics of the harmonic generation, the two mutually coherent harmonic sources should produce a fringe visibility close to 1 : the two sources are said to be "phase locked." In practice, slightly different conditions in producing the two sources lead to a visibility between 0.4 (long trajectory) and 0.9 (short trajectory) at zero delay. Surprisingly we measure a lower fringe visibility in the region associated to the long trajectory in contrast to previous results [8] where similar (visibility around 0.5 ) values were obtained for each contribution. The different phase locking that we measured for the short and the long trajectories may be due to phase matching effects. In particular, the electronic and atomic dispersions in the two focal regions are locally different and this may play a role for on- and off-axis field construction [10]. Consequently the two-source phase locking might be affected differently for each trajectory. However, we should note that this does not affect the following analysis. The coherence time, i.e., the inverse of the spectral width $\Delta_{q}$, is defined as the full width at half maximum (FWHM) of the visibility in Fig. 2. The field in the inner region has a long coherence time (24 fs, $\Delta_{1} \simeq 0.3 \pm 0.05 \mathrm{~nm}$ ), whereas it has a shorter one ( $8 \mathrm{fs}, \Delta_{2} \simeq 0.9 \pm 0.05 \mathrm{~nm}$ ) in the outer region. This confirms the identification of the inner field to the $\tau_{1}$ contribution (small spectral width) and of the outer field to the $\tau_{2}$ contribution (large spectral width). Our conclusions and measured coherence times are in good agreement with Refs. [8,9]. Considering that the spectral width mainly reflects the intrinsic chirp [see Eq. (3)], we get an estimate of the $\alpha_{2} / \alpha_{1}$ ratio of the phase coefficients from the coherence times; we find $\alpha_{2} / \alpha_{1} \simeq 3$. Although this parameter may not directly scale with $\alpha_{2} / \alpha_{1}$ due to the influence of phase matching, we note that the divergence of the field in the outer region is about 3 times larger than in the inner one [24].

\section{B. Spectral and spatial filtering}

We can further evidence the spectral/spatial correlation by introducing a "downstream" filtering of the HHG generated using only one arm of the Michelson interferometer. To this purpose, a motorized adjustable diaphragm is installed in the far-field just before the monochromator. The method is twofold: (i) we filter out the outer region in the spatial profile with the HHG diaphragm, and monitor the spectral profile, and (ii) we filter out the wings of the spectral profile with the variable slit and monitor the spatial profile in the far field. The laser beam is focused $4 \mathrm{~mm}$ after the argon gas jet in order to maximize the long trajectory contribution. The laser energy is varied around $1 \mathrm{~mJ}$ (intensity $\simeq 5 \times 10^{13} \mathrm{~W} \mathrm{~cm}^{-2}$ at focus) in order to minimize self phase modulation of the fundamental pulse via ionization of the gas medium that would induce a blueshift of the laser and subsequently of the harmonic spectra.

Spatial filtering. In the first filtering operation (i), we consider the spatial profile of H17 in Fig. 3(a). It clearly exhibits the inner and outer regions of the inset in Fig. 2, associated to $\tau_{1}$ and $\tau_{2}$ contributions, respectively. Now, we vary the HHG diaphragm from $10 \mathrm{~mm}$ (full beam) to $1 \mathrm{~mm}$. The sig- 

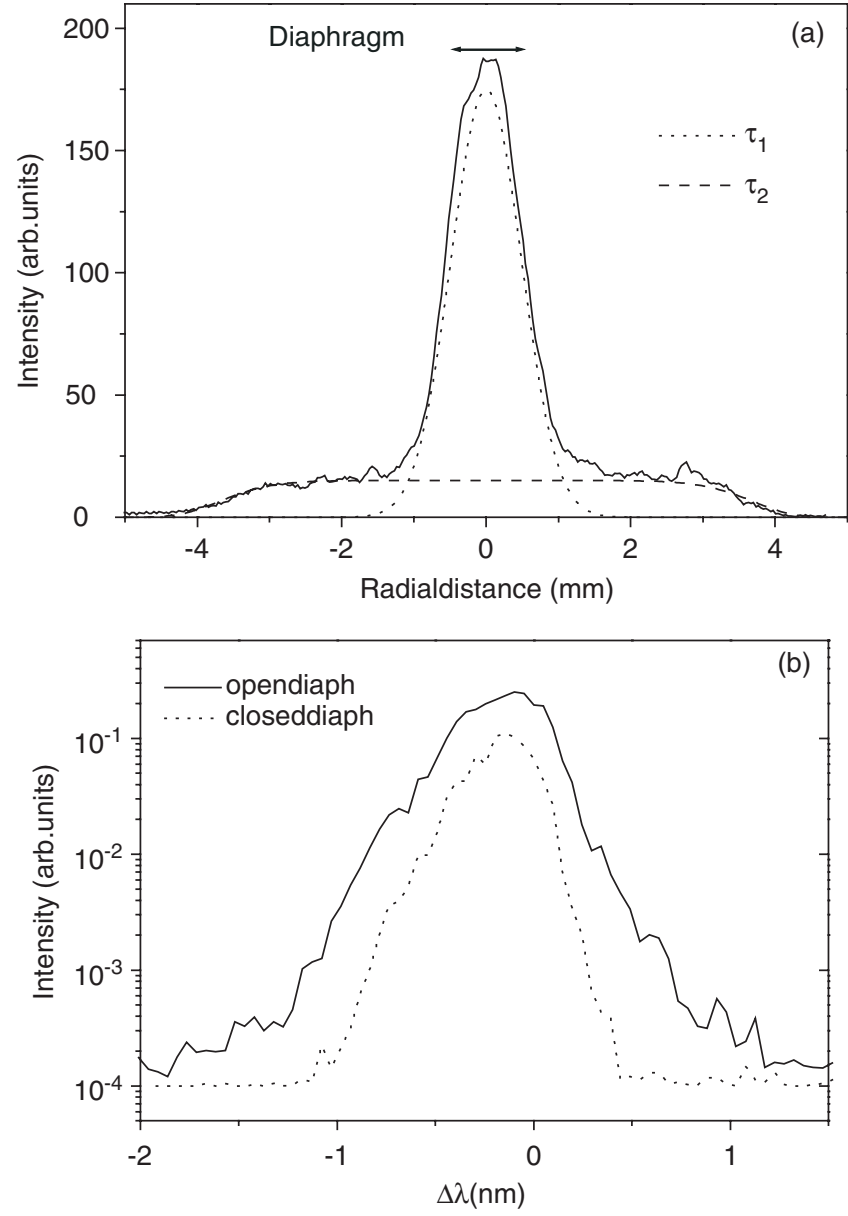

FIG. 3. (a) Spatial profile of the 17th harmonic generated in argon with a laser energy of $0.85 \mathrm{~mJ}$ when the laser is focused $4 \mathrm{~mm}$ after the gas jet. The laser diaphragm is $12.5 \mathrm{~mm}$. Fits of $\tau_{1}$ (Gaussian, dotted line) and $\tau_{2}$ contributions (superGaussian, dashed line). The arrow shows the size of the $1 \mathrm{~mm}$ closed HHG diaphragm. (b) Corresponding spectral profiles for an open and a closed HHG diaphragm.

nal decreases and, more importantly, is spectrally narrowed as shown in Fig. 3(b). We relate this narrowing to a substantial cut of the $\tau_{2}$ contribution operated in the spatial domain.

The evolution of the integrated harmonic signal and of the spectral width (FWHM) as a function of the HHG beam aperture is shown in Fig. 4, for two laser energies 0.85 and $1.25 \mathrm{~mJ}$ with a laser diaphragm of $12.5 \mathrm{~mm}$. The larger widths at $1.25 \mathrm{~mJ}$ reflect the intensity-dependent broadening, $\Delta_{j} \sim \alpha_{j}(\partial I / \partial t)$ due to the intrinsic chirp [10]. For both energies, the maximum values, 0.5 and $0.6 \mathrm{~nm}$ for full $\mathrm{HHG}$ beam aperture, correspond to the profile under which the spectrally narrow $\tau_{1}$ contribution $(\sim 0.3 \mathrm{~nm})$ is superimposed to the dominant and spectrally large $\tau_{2}$ contribution $(\sim 0.9 \mathrm{~nm})$; the resulting effective FWHM is therefore in between those of the two fields. The minimum value, around $0.35 \mathrm{~nm}$ for both energies, is comparable to that of $\tau_{1}$ contribution.

In Fig. 4(a), the integrated signal is compared to the one derived from the spatial profile in Fig. 3(a), simulating numerically the transmission of the HHG diaphragm. In the

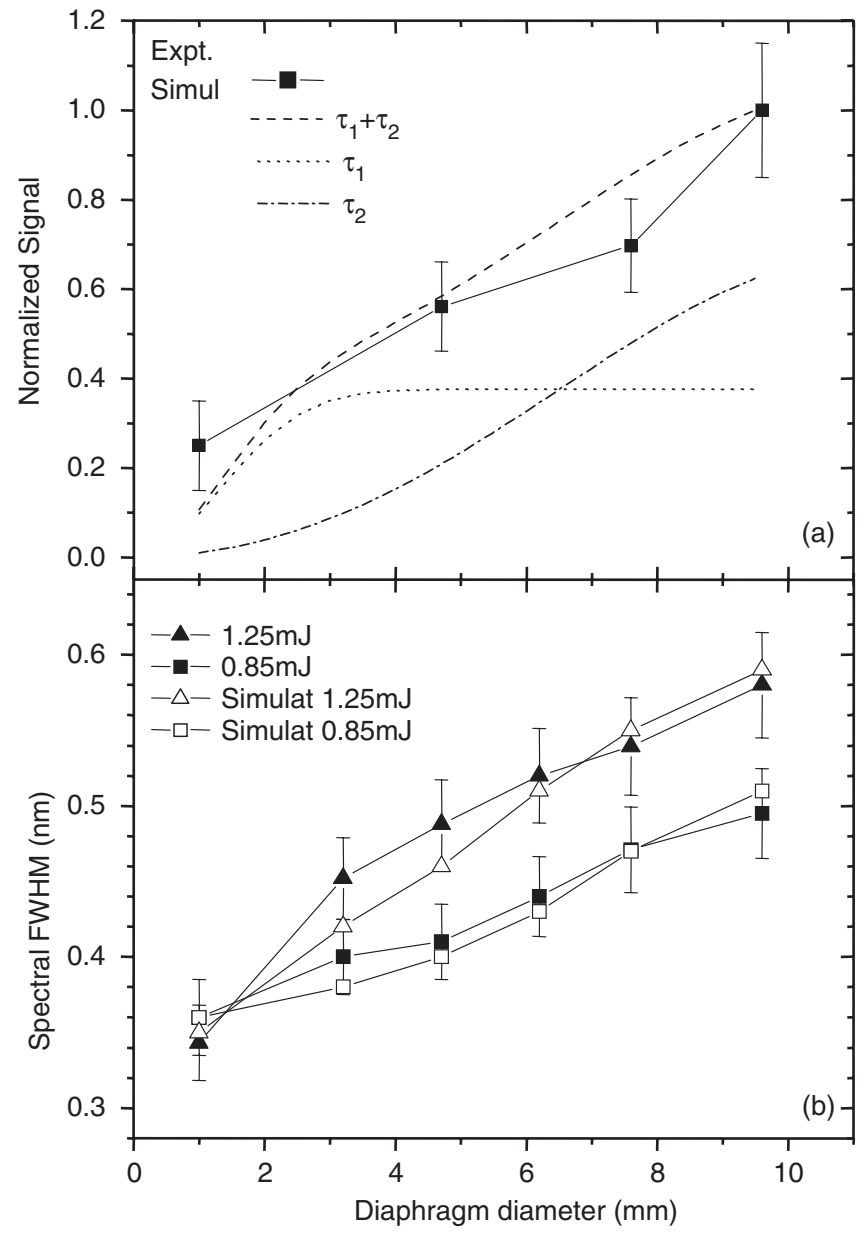

FIG. 4. (a) Integrated spectral intensity of H17 generated in argon as a function of the HHG diaphragm diameter, compared to the simulated signal, sum of the $\tau_{1}$ and $\tau_{2}$ contributions. The $12.5 \mathrm{~mm}$ apertured, $0.85-\mathrm{mJ}$ laser pulse is focused $4 \mathrm{~mm}$ after the gas jet. (b) HHG spectral width for two laser energies; the experimental widths are compared to the effective Gaussian width of the simulated profiles.

latter, we have parametrized the spatial profiles associated to the $\tau_{1}$ (central structure approximated as a Gaussian profile) and $\tau_{2}$ contributions (pedestal approximated as a super Gaussian profile). The intensity of each trajectory contribution corresponds to the integrated signal under each curve simulating the $\tau_{1}$ and $\tau_{2}$ profiles. The long trajectory contribution is 1.6 times larger than the short trajectory contribution for the full beam. Then, we can estimate the energies $E_{1}(d)$ and $E_{2}(d)$, respectively, in the $\tau_{1}$ and $\tau_{2}$ contributions by taking into account their respective divergence extracted from Fig. 3(a). The total energy $E_{1}(d)+E_{2}(d)$ transmitted through the HHG diaphragm of diameter $d$ plotted in Fig. 4(a) compares satisfactorily with the integrated spectral profile; the plot of $E_{1}(d)$ and $E_{2}(d)$ illustrates how the HHG diaphragm changes the relative weight of the two contributions. Now, we want to correlate, at least semiquantitatively, the variation of the $\tau_{1}$ and $\tau_{2}$ contributions to the one of the spectral widths in Fig. 4(b). For this, we assume that for each contribution, the spectral profile can be represented by a Gaussian function $G_{j}(\lambda)$, with width $\Delta_{j}$. We can retrieve the 


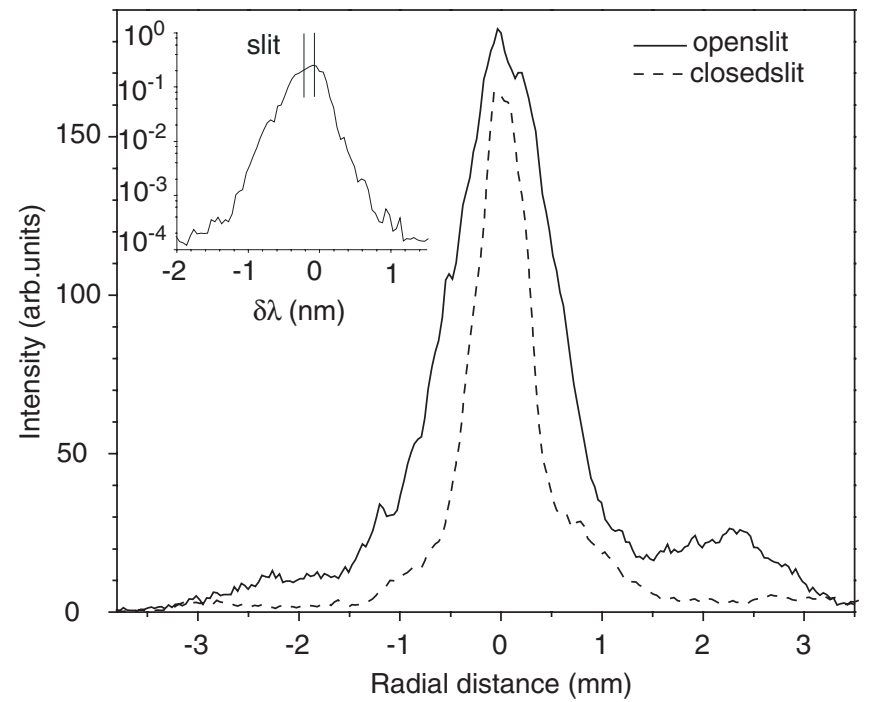

FIG. 5. Spatial profiles of the 17th harmonic generated in argon when the slit in the spectral plane is opened or closed to $\Delta \lambda=0.2 \mathrm{~nm}$. The $1-\mathrm{mJ}$ laser pulse is focused $4 \mathrm{~mm}$ after the gas jet. The inset shows the corresponding spectral profile.

spectral profile of the light filtered, by forming the quantity $\frac{E_{1}(d)}{\Delta_{1}} G_{1}(\lambda)+\frac{E_{2}(d)}{\Delta_{2}} G_{2}(\lambda)$, in which the $G_{j}(\lambda)$ are pondered by the spectral densities $\frac{E_{j}(d)}{\Delta_{j}}$ in each of the $\tau_{j}$ contributions. In the simulation, the widths $\Delta_{i}$ are chosen close to their estimated values in Fig. 2. The effective width (FWHM) of the simulated profile is compared to the measured width at $0.85 \mathrm{~mJ}$ in Fig. 4(b). Similar agreement is obtained for the spectral profile modelization at $1.25 \mathrm{~mJ}$ laser energy. The agreement between measured and simulated widths should be considered as semiquantitative. It evidences the clear correlation between the variation of the spatial and spectral profiles. It allows to assign this correlation to the differential filtering, in the spatial domain, of the $\tau_{1}$ and $\tau_{2}$ contributions. By filtering the outer region of the spatial distribution, we obviously do not "cut" the full $\tau_{2}$ component, but reduce its contribution below $10 \%$ of the total signal.

Spectral filtering. Conversely, in the second filtering operation (ii) on $\mathrm{H} 17$, using a variable slit in the monochromator, we "cut" the spectral distribution and monitor the spatial one. Figure 5 displays the spatial profiles of $\mathrm{H} 17$ for two slit sizes, respectively transmitting the full spectrum (opened slit) and selecting the central width $\Delta \lambda=0.2 \mathrm{~nm}$. For the closed slit, the outer region of the spatial profile is almost completely suppressed, the central structure is narrower.

Symmetrically to filtering (i), we can clearly correlate the wings of the spectral profile to the outer region of the spatial distribution, related to the same field, i.e., the $\tau_{2}$ contribution. In Fig. 5, the reduction of the integrated signal is not as large but still comparable with the one measured in Fig. 4(a) for a small diaphragm aperture.

Finally, the filtering operations (i) and (ii) show that we can, in a consistent way, estimate the weight of the $\tau_{1}$ and $\tau_{2}$ contributions from either the spatial profile of the far field, or the spectral profile. The filtering operation (i) in the spatial domain appears even easier and more efficient than in the
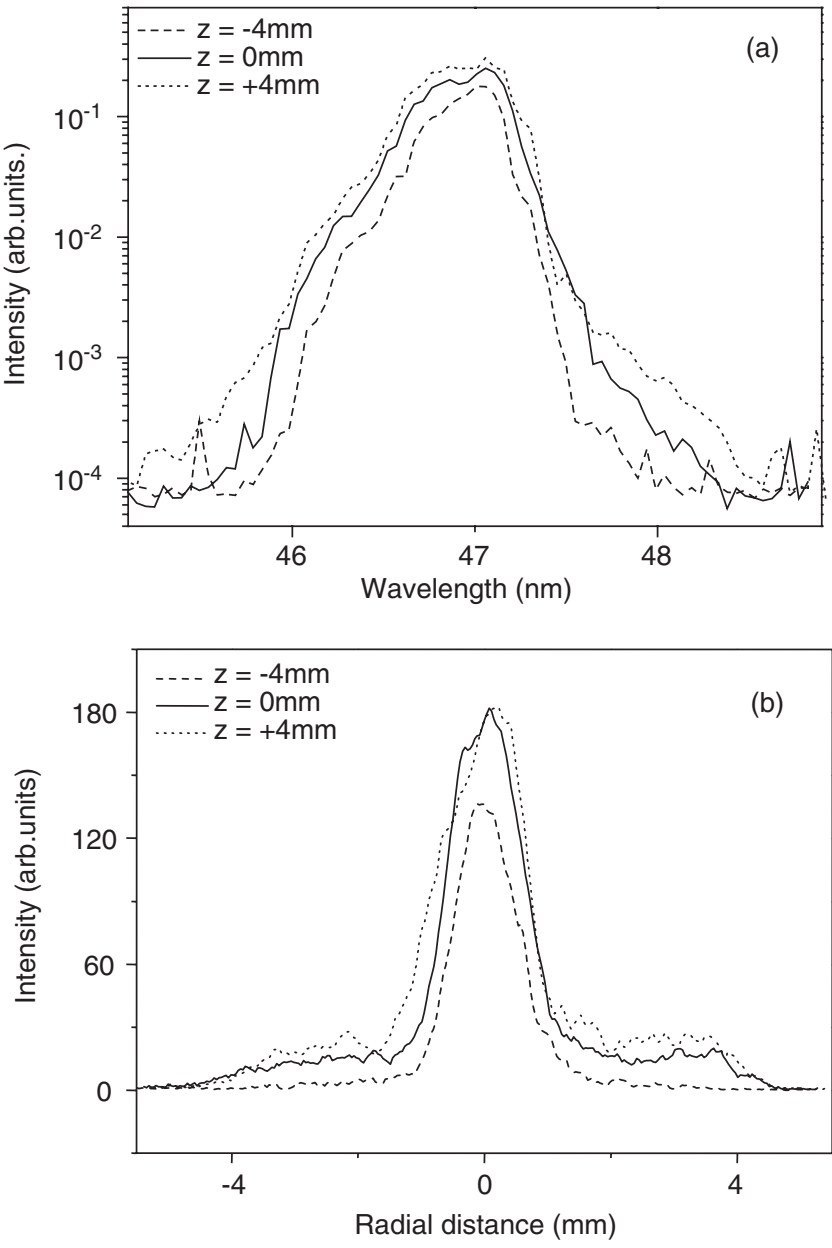

FIG. 6. Measured spectral (a) and spatial (b) profiles of the 17th harmonic as a function of the gas jet position relative to the focus. The laser energy is $1.25 \mathrm{~mJ}$ and the laser diaphragm is $12.5 \mathrm{~mm}$.

spectral one. The experimental evidence and our simple analysis are at least in qualitative agreement with the full simulations by Gaarde et al. [24], where the atomic dipole was calculated from time-dependent Schrödinger equation and propagation fully taken into account.

\section{Variations of the path contributions}

We illustrate now how we can analyze the path contributions when some of the generation parameters are varied. First, we have studied the spectral and spatial profiles of the harmonic emission as a function of the jet/focus position $\left(z=z_{\text {focus }}-z_{\text {jet }}>0\right.$ for focus after the jet $)$. They are, respectively, shown for H17 in Figs. 6(a) and 6(b) (laser energy $E=1.25 \mathrm{~mJ}$, laser diaphragm $d=12.5 \mathrm{~mm}$ ). As observed in the previous studies either in the spatial $[9,41]$ or in the spectral $[10,42]$ domain, we measure a strong dependence of the profiles with the focus position. Our measurement of both quantities allows us to correlate their variation. When the laser is focused before the gas jet $(z=-4 \mathrm{~mm})$, the spectral and spatial profiles are narrow. When the focus moves into $(z=0 \mathrm{~mm})$ and after $(z=+4 \mathrm{~mm})$ the gas jet, the total signal increases, the spectral profile broadens and a pedestal ap- 

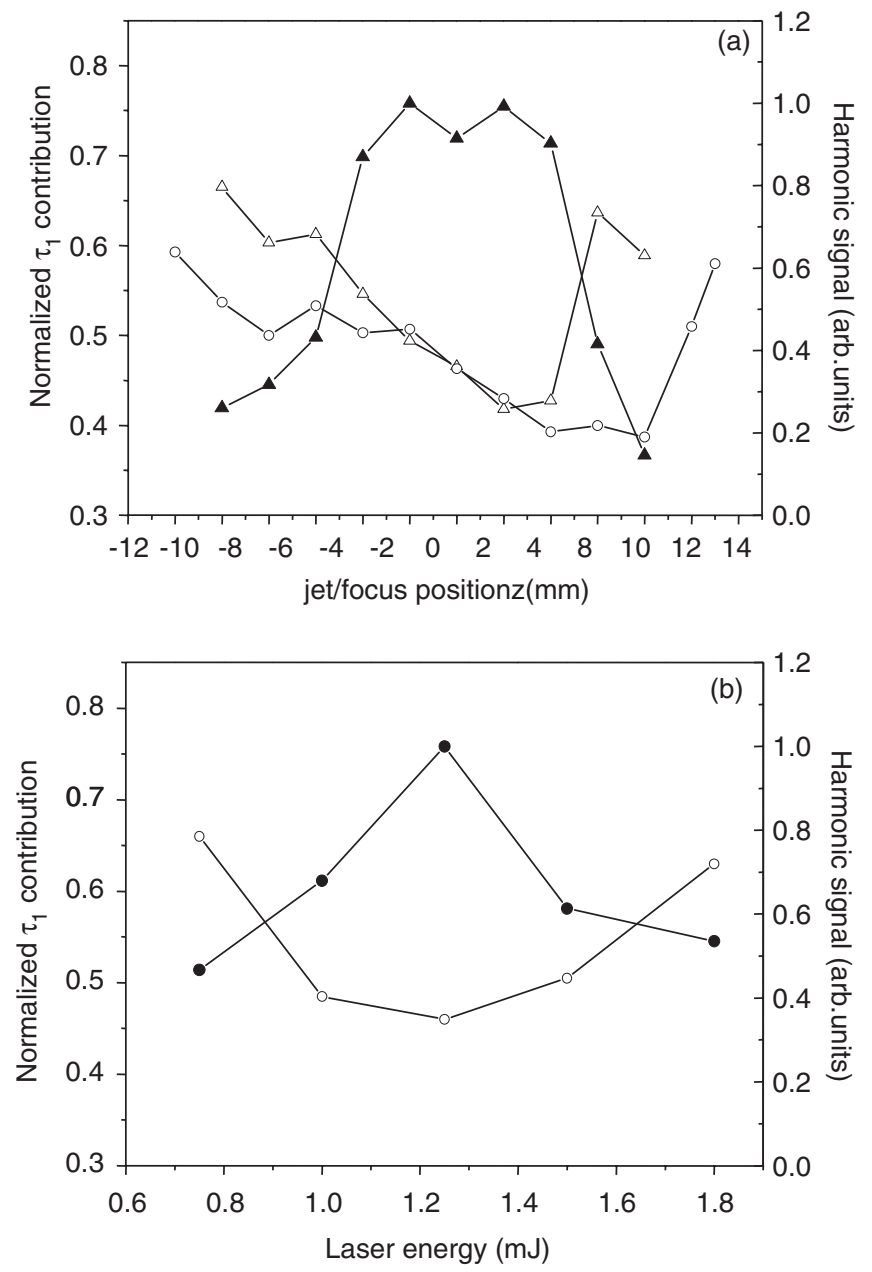

FIG. 7. (a) Contribution of the short trajectory for $\mathrm{H} 17$ as a function of the relative jet-to-focus position $z$ at $E=1.25 \mathrm{~mJ}$ and laser diaphragm $d=12.5 \mathrm{~mm}$ (open circle). The same dependance together with the total harmonic signal are shown for a laser energy of $0.85 \mathrm{~mJ}$ and $d=14 \mathrm{~mm}$ (respectively, open and full triangles). (b) Contribution of the short trajectory as a function of the laser energy at $z=+2 \mathrm{~mm}$ and $d=12.5 \mathrm{~mm}$. The total harmonic signal is plotted in full circle. The error bars (not shown) do not exceed $10 \%$ of the signal.

pears in the spatial distribution. We attribute the broadening of both the spectral and spatial profiles to the onset of $E_{2}$ associated with the $\tau_{2}$ contribution, whereas the central structure is mainly attributed to $E_{1}$ associated with $\tau_{1}$. At large $z>+4 \mathrm{~mm}$, the signal decreases; the profiles are narrow again. The variations of the spectral and spatial profiles are clearly correlated as a function of focusing. First, they reflect how z-dependent phase matching can favor the one or the other contribution: this is the principle of the up-stream control of HHG. Second, for phase-matched emission, they reflect the dependence of the field parameters (chirp) on the laser intensity [10]. A more quantitative analysis is performed in Fig. 7(a), where, together with the integrated signal, we have plotted the relative weight of $\tau_{1}$ contribution (in percentage of total signal) as a function of the jet to focus position $z$. The $\tau_{1}$ weight is obtained from a fit of the $1 \mathrm{D}$ radial profile in Fig. 6(b) as a sum of Gaussian/
superGaussian components [see also Fig. 3(a)], and further $2 \mathrm{D}$ integration. The $\tau_{1}$ contribution is slightly higher when the laser is focused before the gas jet (between $z=-10 \mathrm{~mm}$ and $z=0 \mathrm{~mm}$ ). For $z>0$, the $\tau_{2}$ contribution increases significantly up to $60 \%$ of the total signal. This corresponds to a phase matching achieved in the off-axis region of the gas jet, involving a large radial gradient $\alpha_{j} \vec{\nabla}(I)$ of the laser intensity in Eq. (2), and resulting in an off-axis XUV emission as shown in the spatial profiles. For $z>+10 \mathrm{~mm}$, the phase matching is again realized preferentially on axis for the $\tau_{1}$ contribution. We find the same generic behavior using a larger diaphragm and a lower laser energy [triangles in Fig. 7(a)]. We naturally observe that with smaller confocal parameter and intensity, the harmonic signal varies more rapidly with $z$. Note that the signal dependence indicates that when $\tau_{2}$ component is phase matched, the total signal is maximum.

In Fig. 7(b), we have plotted the weight of the $\tau_{1}$ contribution for $\mathrm{H} 17$ as a function of the laser energy, for $z=+2 \mathrm{~mm}$ and diaphragm $d=12.5 \mathrm{~mm}$. The $\tau_{1}$ contribution slightly dominates at low energy and then decreases when energy increases. When the harmonic total output is optimized $(E=1.25 \mathrm{~mJ}), \tau_{2}$ becomes dominant. Qualitatively, this variation reflects the intensity-dependent phase matching of $\tau_{1}$ and $\tau_{2}$ contributions: the $\tau_{2}$ component is efficiently phase matched for large radial gradient obtained at high intensity. The further increase of $\tau_{1}$ contribution may be attributed to the relative degradation of phase matching in the presence of strong ionization and electronic dispersion, which is more critical off axis (large gradients) for $\tau_{2}$ than it is on axis for $\tau_{1}$. The total signal consequently decreases.

\section{D. "Up-" and "down-stream" filtering of one contribution}

Our analysis shows how control and filtering of the path contributions can be combined, provided that the generation conditions are properly defined. First we can control the quantum path contributions with the energy of the generating laser. Hence, as previously shown in Fig. 7(b), one may favor either the short or the long trajectory by slightly tuning laser energy. Second, we have an additionnal control of quantum path contributions with the focusing geometry. To optimize the $\tau_{1}$ contribution in our geometry, there is advantage to focus the laser close to the gas jet at $z \simeq 0: \tau_{1}$ contributes to $50 \%$ of the total signal which is close to its maximum. Additionnaly we have put a diaphragm to suppress the off-axis signal and we found a $\tau_{1}$ contribution that is around one order of magnitude larger than that of $\tau_{2}$. The measured $E_{1} / E_{2}$ ratio for the full $\mathrm{HHG}$ beam and for the beam apertured at $1 \mathrm{~mm}$ at various focus positions and at two laser energies is reported in Table I. By putting a $\phi=1 \mathrm{~mm}$ diaphragm in the far field, we measure a contribution from the short quantum path that strongly dominates. The above filtering can be used for any harmonic in the plateau region. As a result, almost "pure" $\tau_{1}$ contribution for a set of harmonic components will be obtained. The long trajectory can also be selected. From Fig. 7(b), we note that $\tau_{2}$ emission is maximum $(60 \%$ of the total signal) at $z=+4 \mathrm{~mm}$, where the har- 
TABLE I. Measured ratio $E_{1} / E_{2}$ as a function of jet to focus position at two laser energies. Column A: for the full beam, column $\mathrm{B}$ : for the beam apertured at $\phi=1 \mathrm{~mm}$.

\begin{tabular}{clclc}
\hline \hline \multirow{2}{*}{$\begin{array}{c}\text { Jet to focus } \\
\text { position }(\mathrm{mm})\end{array}$} & $\mathrm{A}$ & $\mathrm{B}$ & $\mathrm{A}$ & $\mathrm{B}$ \\
\cline { 2 - 5 } & $\mathrm{A}$ & 8 & 1.5 & 11 \\
\hline 8 & 0.7 & 9 & 0.7 & 6 \\
4 & 0.8 & 12 & 1 & 9 \\
0 & 1 & 14 & 1.6 & 13 \\
-4 & 1.1 & 14 & 2 & 16 \\
-8 & 1.2 & $14.25 \mathrm{~mJ}$ \\
\hline \hline
\end{tabular}

monic signal is high. We can select $\tau_{2}$ just by putting a disk inside the beam to stop the on-axis emission where $\tau_{1}$ is emitted [43].

This analysis is crucial for the generation of clean and intense attosecond pulses. The cutoff region of the harmonic spectrum, where a single path contribute to the emission, has been used to produce isolated attosecond pulses [28,44]. In order to produce shorter and more intense pulses, the plateau region should be used, and this applies both to trains and to isolated pulses obtained through polarization gating $[34,45-47]$. But the contribution of the two quantum paths, with a relative weight determined both by the atomic dipole and the z-dependent phase matching, directly affects the phase locking between consecutive harmonics [30-32] and blurs the attosecond structure. The generation of a single attosecond burst per half optical cycle thus requires that a single quantum path is selected.

\section{CONCLUSION}

In summary, our measurements demonstrate that we can now have a complete control of the quantum path contributions in high harmonic generation. The two contributions, $\tau_{1}$ and $\tau_{2}$ for, respectively, the short and the long quantum paths, are identified from their strongly correlated properties in the spatial and spectral domains. We have studied them as a function of generation parameter (laser intensity, focus position), emphasizing how phase matching can favor the one or the other contribution. Phase matching therefore serves to the "up-stream" control of the path selection. A simple method of path selection after the generation process or "down-stream" consists in filtering the harmonic emission either in the spatial or in the spectral domain. Short or long quantum path can be, respectively, chosen by adjusting in the far field a diaphragm (on axis emission selected) or a disk (off axis emission selected). This quantum path selection and optimization should lead to the generation of regular and intense attosecond pulses.

\section{ACKNOWLEDGMENTS}

This research was supported by the Marie Curie Research Training Network XTRA (Grant No. MRTN-CT-2003505138), the Integrated Initiative of Infrastructure LASERLAB-EUROPE (Grant No. RII3-CT-2003-506350, FOSCIL) and the New Emerging Science and Technology Contract No. TUIXS (NEST-012843).
[1] P. Salières, A. L'Huillier, Ph. Antoine, and M. Lewenstein, Adv. At., Mol., Opt. Phys. 41, 83 (1999).

[2] T. Brabec and F. Krausz, Rev. Mod. Phys. 72, 545 (2000).

[3] T. Ditmire, E. T. Gumbrell, R. A. Smith, J. W. G. Tisch, D. D. Meyerhofer, and M. H. R. Hutchinson, Phys. Rev. Lett. 77, 4756 (1996).

[4] L. Le Déroff, P. Salières, and B. Carré, Opt. Lett. 23, 1544 (1998).

[5] L. Le Déroff, P. Salières, B. Carré, D. Joyeux, and D. Phalippou, Phys. Rev. A 61, 043802 (2000).

[6] H. Merdji, P. Salières, L. Le Déroff, J.-F. Hergott, B. Carré, D. Joyeux, D. Descamps, J. Norin, C. Lyngå, A. L'Huillier, C. G. Wahlström, M. Bellini, and S. Hüller, Laser Part. Beams 18, 495 (2000).

[7] R. Bartels, A. Paul, H. Green, H. C. Kapteyn, M. M. Murnane, S. Backus, I. P. Christov, Y. W. Liu, D. Attwood, and C. Jacobsen, Science 297, 376 (2002).

[8] M. Bellini, C. Lyngå, A. Tozzi, M. B. Gaarde, T. W. Hänsch, A. L'Huillier, and C. G. Wahlström, Phys. Rev. Lett. 81, 297 (1998).

[9] C. Lyngå, M. B. Gaarde, C. Delfin, M. Bellini, T. W. Hänsch, A. L'Huillier, and C. G. Wahlström, Phys. Rev. A 60, 4823 (1999).

[10] P. Salières, B. Carré, L. Le Déroff, F. Grasbon, G. G. Paulus,
H. Walther, R. Kopold, W. Becker, D. B. Milosevic, A. Sanpera, and M. Lewenstein, Science 292, 902 (2001).

[11] J. Mauritsson, P. Johnsson, R. López-Martens, K. Varju, W. Kornelis, J. Biegert, U. Keller, M. B. Gaarde, K. J. Schafer, and A. L'Huillier, Phys. Rev. A 70, 021801 (2004).

[12] J.-F. Hergott, M. Kovačev, H. Merdji, C. Hubert, Y. Mairesse, E. Jean, P. Breger, P. Agostini, B. Carré, and P. Salières, Phys. Rev. A 66, 021801 (2002).

[13] E. Takahashi, Y. Nabekawa, and K. Midorikawa, Opt. Lett. 27, 1920 (2002).

[14] P. Salières, L. Le Déroff, T. Auguste, P. Monot, P. d'Oliveira, D. Campo, J. F. Hergott, H. Merdji, and B. Carré, Phys. Rev. Lett. 83, 5483 (1999).

[15] D. Descamps, C. Lyngå, J. Norin, A. L'Huillier, C. G. Wahlström, J. F. Hergott, H. Merdji, P. Salières, M. Bellini, and T. W. Hänsch, Opt. Lett. 25, 135 (2000).

[16] A. L'Huillier, A. Johansson, J. Norin, J. Mauritsson, and C.-G. Wahlström, Eur. Phys. J. D 26, 91 (2003).

[17] P. Zeitoun, G. Faivre, S. Sebban S, T. Mocek, A. Hallou, M. Fajardo, D. Aubert, P. Balcou, F. Burgy, D. Douillet, S. Kazamias, G. de Lacheze-Murel, T. Lefrou, S. le Pape, P. Mercere, H. Merdji, A. S. Morlens, J. P. Rousseau, and C. Valentin, Nature (London) 431, 426 (2004).

[18] D. Garzella, T. Hara, B. Carré, P. Salières, T. Shintake, H. 
Kitamura, and M. E. Couprie, Nucl. Instrum. Methods Phys. Res. A 528, 502 (2004).

[19] P. Agostini and L. F. DiMauro, Rep. Prog. Phys. 67, 813 (2004)

[20] P. B. Corkum, Phys. Rev. Lett. 71, 1994 (1993).

[21] K. J. Schafer, B. Yang, L. F. Dimauro, and K. C. Kulander, Phys. Rev. Lett. 70, 1599 (1993).

[22] M. Lewenstein, P. Balcou, M. Y. Ivanov, A. L'Huillier, and P. B. Corkum, Phys. Rev. A 49, 2117 (1994).

[23] K. C. Kulander, in Super-Intense Laser-Atom Physics, edited by B. Piraux, A. L'Huillier, and K. Rzazewski, Vol. 316 of NATO Advanced Science Institute, Ser. B: Physics (Plenum, New York, 1994), p. 95.

[24] M. B. Gaarde, F. Salin, E. Constant, P. Balcou, K. J. Schafer, K. C. Kulander, and A. L'Huillier, Phys. Rev. A 59, 1367 (1999).

[25] M. B. Gaarde and K. J. Schafer, Phys. Rev. A 65, 031406 (2002).

[26] Y. Mairesse, A. de Bohan, L. J. Frasinski, H. Merdji, L. C. Dinu, P. Monchicourt, P. Bréger, M. Kovačev, R. Taieb, B. Carré, H. G. Muller, P. Agostini, and P. Salières, Science 302, 1540 (2003).

[27] P. M. Paul, E. S. Toma, P. Bréger, G. Mullot, F. Augé, P. Balcou, H. G. Muller, and P. Agostini, Science 292, 1689 (2001).

[28] R. Kienberger, E. Goulielmakis, M. Überacker, A. Baltuška, V. Yakovlev, F. Bammer, A. Scrinzi, T. Westerwalbesloh, U. Kleineberg, U. Heinzmann, M. Drescher, and F. Krausz, Nature (London) 427, 817 (2004).

[29] P. Salières, A. L'Huillier, and M. Lewenstein, Phys. Rev. Lett. 74, 3776 (1995).

[30] P. Antoine, A. L'Huillier, and M. Lewenstein, Phys. Rev. Lett. 77, 1234 (1996).

[31] M. B. Gaarde and K. J. Schafer, Phys. Rev. Lett. 89, 213901 (2002).

[32] Y. Mairesse, A. de Bohan, L. J. Frasinski, H. Merdji, L. C. Dinu, P. Monchicourt, P. Bréger, M. Kovačev, T. Auguste, B. Carré, H. G. Muller, P. Agostini, and P. Salières, Phys. Rev.
Lett. 93, 163901 (2004).

[33] R. López-Martens, K. Varjù, P. Johnsson, J. Mauritsson, Y. Mairesse, P. Salières, M. B. Gaarde, K. J. Schafer, A. Persson, S. Svanberg, C. G. Wahlström, and A. L'Huillier, Phys. Rev. Lett. 94, 033001 (2005).

[34] E. Constant et al. (unpublished).

[35] L. C. Dinu, H. G. Muller, S. Kazamias, G. Mullot, F. Augé, P. Balcou, P. M. Paul, M. Kovačev, P. Bréger, and P. Agostini, Phys. Rev. Lett. 91, 063901 (2003).

[36] S. A. Aseyev, Y. Ni, L. J. Frasinski, H. G. Muller, and M. Vrakking, Phys. Rev. Lett. 91, 223902 (2003).

[37] K. T. Kim, C. M. Kim, M. G. Baik, G. Umesh, and C. H Nam, Phys. Rev. A 69, 051805 (2004).

[38] Ph. Balcou, P. Salières, A. L'Huillier, and M. Lewenstein, Phys. Rev. A 55, 3204 (1997).

[39] H. Merdji, J. F. Hergott, M. Kovačev, E. Priori, P. Salières, and B. Carré, Laser Part. Beams 22, 275 (2004).

[40] M. Kovačev, S. V. Fomichev, E. Priori, Y. Mairesse, H. Merdji, P. Monchicourt, P. Bréger, J. Norin, A. Persson, A. L'Huillier, C. G. Wahlström, B. Carré, and P. Salières, Phys. Rev. Lett. 95, 223903 (2005).

[41] P. Salières, T. Ditmire, M. D. Perry, A. L'Huillier, and M. Lewenstein, J. Phys. B 29, 4771 (1996).

[42] D. G. Lee, H. J. Shin, Y. H. Cha, K. H. Hong, J. H. Kim, and C. H. Nam, Phys. Rev. A 63, 021801 (2001).

[43] G. Sansone et al., Phys. Rev. A (to be published).

[44] M. Hentschel, R. Kienberger, C. Spielmann, G. A. Reider, N. Milošević, T. Brabec, P. B. Corkum, U. Heinzmann, M. Drescher, and F. Krausz, Nature (London) 414, 509 (2001).

[45] M. Kovačev, Y. Mairesse, E. Priori, H. Merdji, O. Tcherbakoff, P. Monchicourt, P. Bréger, E. Mével, E. Constant, P. Salières, B. Carré, and P. Agostini, Eur. Phys. J. D 26, 7982 (2003).

[46] O. Tcherbakoff, E. Mével, D. Descamps, J. Plumridge, and E. Constant, Phys. Rev. A 68, 043804 (2003).

[47] Y. Mairesse, O. Gobert, P. Bréger, H. Merdji, P. Meynadier, P. Monchicourt, M. Perdrix, P. Salières, and B. Carré, Phys. Rev. Lett. 94, 173903 (2005). 